\title{
Diacronie
}

Studi di Storia Contemporanea

$N^{\circ} 23,3$ | 2015

Mediterraneo cosmopolita

\section{Chiara Giorgi, L'Africa come carriera. Funzioni e funzionari del colonialismo italiano}

\section{Michele Pandolfo}

\section{Q OpenEdition}

Edizione digitale

URL: http://journals.openedition.org/diacronie/2403

DOI: 10.4000/diacronie. 2403

ISSN: 2038-0925

Editore

Association culturelle Diacronie

Notizia bibliografica digitale

Michele Pandolfo, «Chiara Giorgi, L'Africa come carriera. Funzioni e funzionari del colonialismo italiano », Diacronie [Online], № 23, 3 | 2015, documento 6, Messo online il 29 octobre 2015, consultato il 24 septembre 2020. URL : http://journals.openedition.org/diacronie/2403; DOI : https://doi.org/10.4000/ diacronie. 2403 


\section{RECENSIONE:}

\section{Chiara GIORGI, L'Africa come carriera. Funzioni e funzionari del colonialismo italiano, Roma, Carocci, 2012, 222 pp.}

a cura di Michele PANDOLFO *

Il saggio di Chiara Giorgi ${ }^{1}$ intitolato L'Africa come carriera. Funzioni e funzionari del colonialismo italianoaffronta un interessante argomento lasciato ancora ai margini nel panorama ormai vasto e consolidato degli studi accademici sul colonialismo italiano: quello della burocrazia coloniale formata dai pubblici ufficiali che operarono durante le diverse fasi del dominio italiano in Africa² ${ }^{2}$

Questo studio approfondisce con particolare interesse la figura del funzionario coloniale, il suo ruolo all'interno dell'apparato amministrativo delle colonie e il suo apporto alla costruzione del contesto coloniale italiano. La pubblicazione di Giorgi cerca inoltre di abbattere l'anonimato che ha nascosto le esperienze umane e professionali degli italiani mandati in Africa per costruire la macchina burocratica della sfera coloniale, a partire dalla prima colonia italiana: l'Eritrea. Il discorso sui funzionari coloniali diventa di conseguenza molto importante per diverse ragioni: innanzitutto gli studi storici italiani soffrivano di un ritardo cronico su questo argomento rispetto alle

\footnotetext{
${ }^{1}$ Chiara Giorgi è ricercatrice di Storia delle istituzioni politiche presso la facoltà di Scienze politiche dell'Università degli Studi di Genova. Oltre a numerose opere di saggistica e altre curatele si segnalano le due monografie che precedono la presente pubblicazione: GIORGI, Chiara, La sinistra alla Costituente. Per una storia del dibattito istituzionale, Roma, Carocci, 2001; ID., La previdenza del regime. Storia dell'INPS durante il fascismo, Bologna, Il Mulino, 2004.

${ }^{2}$ La bibliografia sul colonialismo italiano è ormai vastissima e comprende molti studi italiani e stranieri che hanno analizzato con diversi approcci questa tematica. Ricordo soltanto alcuni testi fondamentali: DEL BOCA, Angelo, Gli italiani in Africa Orientale, Roma-Bari, Laterza, 19761982; ID., L'Africa nella coscienza degli italiani: miti, memorie, errori, sconfitte, Roma-Bari, Laterza, 1992; ID., Italiani brava gente? Un mito duro a morire, Vicenza, Neri Pozza, 2005, GOGLIA, Luigi, GRASSI, Fabio, Il colonialismo italiano da Adua allimpero, Roma-Bari, Laterza, 1993; LABANCA, Nicola, Oltremare: storia dell'espansione coloniale italiana, Bologna, Il Mulino, 2002. Si aggiunge inoltre lo studio in lingua inglese di BEN-GHIAT, Ruth, FULLER, Mia, Italian Colonialism, New York, Palgrave, 2005.
} 
storiografie degli altri paesi europei; in secondo luogo capire chi sono stati gli uomini andati in Africa per fondare e amministrare i territori conquistati diventa rilevante per unire un ulteriore tassello al complesso quadro della storia coloniale italiana. Gli obiettivi di questo lavoro di ricerca vengono citati dalla stessa autrice nelle prime pagine del testo:

Questo studio muove dall'intento di indagare chi e quanti furono coloro i quali, a più riprese, lavorarono e vissero nelle colonie, governandole e soprattutto quale attività vi svolsero in qualità di amministratori. Ci si è inoltre domandati quali furono le carriere specificamente coloniali che si vennero a creare; e ancor più da dove provenivano sotto il profilo sociale, intellettuale e culturale i funzionari coloniali; in che modo venivano selezionati e reclutati; che cosa li spingeva a questa scelta e come interpretarono la propria parte di colonizzatori di un mondo ritenuto "inferiore", "incivile" e "selvaggio", incarnando la propria presunta "missione" di civilizzazione. Muovendo da questi interrogativi, la ricerca tenta di ricostruire le vicende di questo contingente, soffermandosi in particolare sul personale che ricoprì i gradi più alti dell'amministrazione coloniale3.

Partendo da un iniziale confronto con i modelli dei funzionari francesi e inglesi, il testo mette in luce la contraddittorietà del caso italiano. Se da una parte, infatti, l'Italia ha sempre dimostrato - anche prima dell'unificazione nazionale - un forte interesse per l'Africa, d'altro canto risulta evidente, sin dai primi passi del colonialismo italiano, la mancata organizzazione dei vari poteri dello stato nelle terre africane. Il quadro dell'amministrazione giuridica e civile delle colonie è sempre stato delineato in maniera provvisoria e frammentata, giustificato in parte dal fatto che le nuove acquisizioni coloniali sono state poche e discontinue nel tempo.

Nelle prime colonie del Corno d'Africa, Eritrea e Somalia, venne mandato esclusivamente del personale militare che assolse per diverso tempo anche delle funzioni civili, aspettando l'approvazione di una legislazione adatta a organizzare la nuova situazione coloniale. Soltanto in pochi casi i pubblici ufficiali provenivano da alcuni organismi ministeriali della madrepatria e possedevano una preparazione di carattere amministrativo e giuridico. Ciò che differenzia questo gruppo minoritario di funzionari rispetto ai loro colleghi europei è il fatto che quelli italiani non avevano, quindi, una formazione specifica che li preparasse a svolgere il loro servizio nell'ambiente coloniale. Sicuramente una della motivazioni principali che spinse molti

3 GIORGI, Chiara, L'Africa come carriera. Funzioni e funzionari del colonialismo italiano, Roma, Carocci, 2012, pp. 15-16. 
funzionari a partire per le colonie italiane è stata quella di ottenere un aumento dei propri emolumenti, insieme ad altre indennità vantaggiose per le rispettive carriere. A questo si unì, ma soltanto per pochi, una reale volontà di conoscere il continente africano, che era rappresentato come selvaggio, inospitale ma ricco di risorse.

Ciò che si evince, insomma, dalla peculiarità del caso italiano è che, almeno per tutta la prima fase coloniale, cioè quella del periodo liberale, è completamente assente nella madrepatria una formazione specifica per chi andrà a governare i possedimenti italiani d'Oltremare, a differenza di altri paesi dove vi erano istituzioni predisposte all'istruzione degli agenti coloniali, come per esempio le scuole coloniali in Francia e le altre istituzioni universitarie in Gran Bretagna. Quella italiana invece diventa, nella maggior parte dei casi, un'esperienza diretta che porta a una formazione pragmatica del sapere coloniale.

Durante il periodo liberale, alcune riforme vennero progressivamente promosse e cercarono di regolare il reclutamento dei funzionari coloniali. Tuttavia, la riforma strutturale più rilevante in questo settore venne approvata solo nel 1928, durante il ventennio fascista, quando si giunse a una riorganizzazione definita e completa del settore. Infatti, in tale normativa venne riordinato l'organico dell'amministrazione coloniale confermando, secondo lo schema della riforma della pubblica amministrazione già emanata nel 1923, la suddivisione del personale in tre grandi gruppi - personale direttivo, ausiliario, e di ordine - al quale si applicavano le norme proprie della pubblica amministrazione della madrepatria. Secondo questa nuova riforma le funzioni politiche e amministrative erano affidate al personale coloniale in possesso di una formazione adeguata, escludendo di fatto gli elementi estranei alla sfera africana. L'unica eccezione era riservata alla funzione del governatore, alla quale potevano accedere anche personalità che nella madrepatria già ricoprivano importanti incarichi politici.

Tale importante passaggio nella storia dell'amministrazione coloniale viene così descritto dall'autrice:

Il contingente di uomini partito alla volta dell'Africa a metà degli anni Trenta raggiunge cifre elevatissime ed è nutrito di una ideologia razzista e conquistatrice sempre più dominante. Giovani, senza pregressi di carriera nell'amministrazione, per lo più ex combattenti nelle "terre dell'impero", i nuovi funzionari sono alla ricerca di uno sbocco professionale che li valorizzi. Tuttavia è proprio il regime a scontrarsi con un'evidente contraddizione, frutto delle sue stesse modalità di governo: si tratta della necessità, ai fini della tenuta dell'impero, di avere a 
disposizione funzionari preparati, che abbiano dimestichezza con i luoghi e le popolazioni dominate.

Nel tentativo di conciliare efficienza amministrativa della periferia africana e controllo totalitario, governo d'eccezione e duratura tutela dell'ordine imposto, dominio e conoscenza (l'una strumentale all'altro), il fascismo è spesso costretto a ricorrere agli uomini della cosiddetta "vecchia guardia". Incapace di dar vita a istituzioni di formazione per il personale coloniale, analoghe a quelle caratterizzanti l'esperienza inglese e francese, il regime si avvale del bagaglio di competenze professionali, di contatti e di esperienze propri dell'età liberale, contemporaneamente esasperando e riformulando in termini essenzialisti ed immutabili la distanza che deve separare colonizzatori e colonizzati, la segregazione degli uni rispetto agli altri.

Un altro merito della ricerca proposta in questa pubblicazione è quello di scoprire alcune delle figure appartenute alle schiere degli impiegati pubblici che sono partiti per le colonie africane e lì hanno svolto molti anni di servizio ${ }^{5}$. Tra i percorsi biografici riportati alla luce in queste pagine quello più originale riguarda il caso del funzionario coloniale Dante Odorizzi (1867-1917). Originario di Mantova, Odorizzi entrò in servizio nell'amministrazione civile eritrea alla fine dell'Ottocento, svolgendo a partire dal 1904 importanti funzioni in alcuni Commissariati regionali della colonia fino al 1917, anno del suo tragico suicidio. Dopo l'improvvisa morte del funzionario, al momento dell'apertura del testamento, si scoprì che egli aveva nominato quale erede del suo patrimonio un indigeno di nome Osman Mohamed, con il quale, oltre a essere il suo personale interprete, aveva costruito una relazione sentimentale, già nota nell'ambiente coloniale. Il testamento verrà in seguito dichiarato nullo per la presunta infermità mentale dello stesso Odorizzi, ma questo particolare episodio dimostra quanto ancora poco si sappia effettivamente delle esperienze personali degli italiani che vissero in Africa.

La parabola dei funzionari coloniali continuò anche dopo la fine del dominio italiano in Africa: terminata la seconda guerra mondiale e stabilito il completo ritiro dell'Italia dal continente africano, escludendo l'eccezionalità del mandato fiduciario

\footnotetext{
4 Ibidem, p. 17.

${ }^{5}$ Le figure dei funzionari del colonialismo italiano non sono mai state generalmente oggetto di studi e ricerche. Soltanto alcune personalità rilevanti, come per esempio il governatore dell'Eritrea Ferdinando Martini (1841-1928) e pochi altri, si impegnarono nell'effettiva conoscenza delle culture indigene e nell'apprendimento delle lingue locali. L'ultima ristampa disponibile di una delle opere più conosciute di Ferdinando Martini è la seguente: MARTINI, Ferdinando, Nell'Affrica Italiana, Milano, La biblioteca del Touring Club Italiano, 1998. Stanno emergendo però negli ultimi anni delle pubblicazioni dove vengono raccontate altre biografie di pubblici ufficiali che operarono in Africa durante gli anni del colonialismo.
} 
sulla Somalia, i vari burocrati delle ex colonie italiane tornarono nella madrepatria e vennero reinseriti in altre amministrazioni pubbliche. Smantellato ufficialmente l'apparato statale nelle colonie, la burocrazia coloniale continuò invece la propria esistenza in Italia, ripetendo quella continuità di uomini e strutture che è sempre stata tipica del carattere nazionale italiano. Per questi motivi raccontare le biografie e le mansioni svolte in colonia dai pubblici ufficiali significa comprendere come il loro operato, una volta rientrati in patria, abbia influenzato anche l'identità della nazione italiana, visto che l'Italia ha raggiunto la propria unità nazionale più tardi di altri paesi europei.

La stessa autrice sottolinea l'importanza di conoscere tutte quelle esperienze biografiche che aiutino a comprendere quali furono gli strumenti di governo adottati dai vari funzionari e come essi influirono concretamente nell'effettiva amministrazione e distribuzione del potere coloniale, soprattutto nei rapporti con le popolazioni dominate:

\begin{abstract}
Furono i funzionari a decidere in molti casi delle sorti dei colonizzati, a tracciare le unità amministrative delle colonie mutando equilibri preesistenti, a rimodellare la realtà sociale, politica e culturale locale, ad impiantare direttamente modelli istituzionali propri della madrepatria e del tutto estranei al contesto dell'Africa, riadattandoli di volta in volta alle esigenze del dominio e del controllo dei territori e delle popolazioni. Furono i funzionari a riorganizzare i diversi territori governati, frammentandoli e disponendoli secondo nuove unità, con operazioni volte a creare, anche inventando, nuovi gruppi etnici, a manipolare, esasperandole, diverse tradizioni e identità locali, in uno scambio mutuo di consegne fra l'amministrazione e l'etnografia ${ }^{6}$.
\end{abstract}

Il testo di Chiara Giorgi, che si basa su accurate ricerche d'archivio svolte sia in Italia che all'estero, si conclude con una buona bibliografia sul tema generale del colonialismo italiano e, nello specifico, proponendo anche alcuni titoli di giurisprudenza coloniale. Queste indicazioni bibliografiche risultano essere un utile strumento di ricerca sia per studiosi che si approccino a tali argomenti per la prima volta sia per quelli che desiderino svolgere ulteriori approfondimenti sulla materia specifica.

${ }^{6}$ GIORGI, Chiara, L'Africa come carriera. Funzioni e funzionari del colonialismo italiano, cit., p. 201. 


\section{* L'autore}

Michele Pandolfo ha conseguito la Laurea Specialistica in Filologia Moderna; è oggi Dottorando in Storia Culture e Strutture delle Aree di Frontiera, Università degli Studi di Udine, in cotutela con l’Université Paul Valery di Montpellier.

URL: < http://www.studistorici.com/progett/autori/\#Pandolfo >

\section{Per citare questo articolo:}

PANDOLFO, Michele, «Recensione: Chiara GIORGI, L'Africa come carriera. Funzioni e funzionari del colonialismo italiano, Roma, Carocci, 2012, 222 pp.», Diacronie. Studi di Storia Contemporanea : Mediterraneo cosmopolita: le relazioni culturali tra Turchia ed Europa, 29/10/2015,

URL:< http://www.studistorici.com/2015/10/29/pandolfo_numero_23/ >

Diacronie Studi di Storia Contemporanea $\beta$ ww.diacronie.it

Risorsa digitale indipendente a carattere storiografico. Uscita trimestrale. redazione.diacronie@hotmail.it

Comitato di redazione: Jacopo Bassi - Luca Bufarale - Elisa Grandi - Deborah Paci - Fausto Pietrancosta - Matteo Tomasoni - Luca Zuccolo 